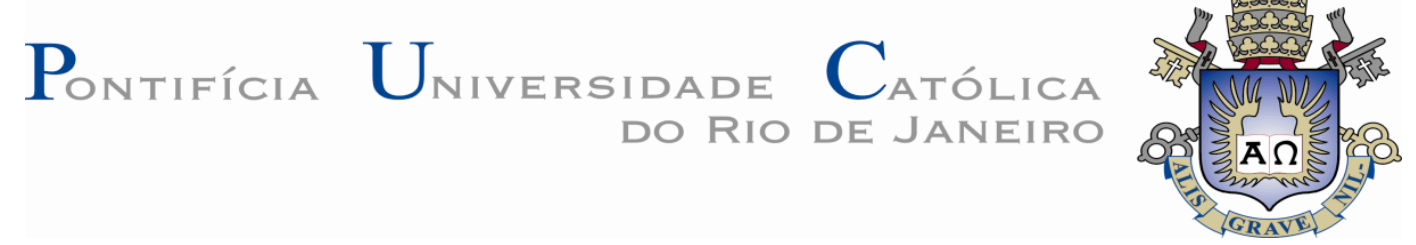

Fabio Paula Brum

Modelo de Transferência de Massa Convectivo para Predição de Depósito de Parafina em Escoamento

Multifásico

Dissertação de Mestrado

Dissertação apresentada ao Programa de PósGraduação em Engenharia Mecânica da PUC-Rio como requisito parcial para obtenção do título de Mestre em Engenharia Mecânica.

Orientadora: Profa. Angela Ourivio Nieckele Co-Orientador: Prof. Sidney Stuckenbruck

Rio de Janeiro

Setembro de 2014 


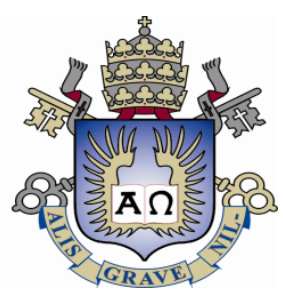

Fabio Paula Brum

\title{
Modelo de Transferência de Massa Convectivo para Predição de Depósito de Parafina em Escoamento \\ Multifásico
}

\begin{abstract}
Dissertação apresentada como requisito parcial para obtenção do grau de Mestre pelo Programa de Pósgraduação em Engenharia Mecânica do Departamento de Engenharia Mecânica do Centro Técnico Cientifico da PUC-Rio. Aprovada pela Comissão Examinadora abaixo assinada.
\end{abstract}

Profa. Angela Ourivio Nieckele

Orientadora

Departamento de Engenharia Mecânica -PUC- Rio

Prof. Sidney Stuckenbruck

Co-orientador

Olympus Software Científico e Engenharia

Prof. Paulo Roberto de Souza Mendes

Departamento de Engenharia Mecânica -PUC- Rio

Dr. João Neuenschwander Escosteguy Carneiro Instituto SINTEF do Brasil

Prof. José Eugenio Leal

Coordenador Setorial do Centro

Técnico Científico - PUC-Rio

Rio de Janeiro, 11 de Setembro de 2014 
Todos os direitos reservados. É proibida a reprodução total ou parcial do trabalho sem autorização da universidade, do autor e do orientador.

\section{Fabio Paula Brum}

Graduou-se em Engenharia Naval no Depto. de Engenharia Oceânica UFRJ (Universidade Federal do Rio de Janeiro), em 1996. É Engenheiro de Petróleo na PETROBRAS, onde atua na área de Elevação e Escoamento.

Ficha Catalográfica

Brum, Fabio Paula

Modelo de transferência de massa convectivo para predição de depósito de parafina em escoamento multifásico / Fabio Paula Brum; orientador: Angela Ourivio Nieckele; co-orientador: Sidney Stuckenbruck. - 2014.

111 f.: il. (color.) ; $30 \mathrm{~cm}$

Dissertação (mestrado)-Pontifícia Universidade Católica do Rio de Janeiro, Departamento de Engenharia Mecânica, 2014. Inclui bibliografia

1. Engenharia Mecânica - Teses. 2. Deposição de Parafina. 3. Convecção. 4. Escoamento Multifásico. 5. Simulação Numérica. 5. Unidimensional. 6. Modelo de Deslizamento. I. Nieckele, Angela Ourivio. II. Stuckenbruck, Sidney. III. Pontifícia Universidade Católica do Rio de Janeiro. Departamento de Engenharia Mecânica. IV. Título. 


\section{Agradecimentos}

A minha esposa e filhos pelo amor, compreensão e apoio durante todo o período deste projeto de vida.

Aos meus pais e irmão que me proporcionaram uma estrutura familiar e a melhor orientação para que eu obtivesse mais esta conquista, minha eterna gratidão.

A Professora Angela Ourivio Nieckele, minha orientadora, pela credibilidade, paciência, disponibilidade e capacidade de ensino demonstrada neste perído de trabalho em conjunto.

Ao orientador, o Professor Sidney Stuckenbruck, pela amizade, orientação e apoio no desenvolvimento deste trabalho, cuja base, o aplicativo Transflux, é de sua autoria.

Aos professores da PUC-Rio pelo ensino excelente, e pelos conhecimentos adquiridos.

A empresa Petrobras, por me indicar para realização deste mestrado e pelo apoio ao longo de todo período do curso.

Aos meus colegas da UO-RIO/IPP/EES pela ajuda na obtenção das informações utilizadas neste trabalho e pelo incentivo na conclusão do curso.

A todos os meus familiares e amigos que sempre me incentivaram e acreditaram no meu sucesso. 


\section{Resumo}

Brum, Fabio Paula; Nieckele, Angela Ourivio; Stuckenbruck, Sidney. Modelo de Transferência de Massa Convectivo para Predição de Depósito de Parafina em Escoamento Multifásico. Rio de Janeiro, 2014. 111p. Dissertação de Mestrado - Departamento de Engenharia Mecânica, Pontifícia Universidade Católica do Rio de Janeiro.

Óleos brutos altamente parafínicos podem causar problemas operacionais significativos, tais como bloqueio de um oleoduto devido à precipitação e deposição de componentes de parafina durante a produção e transporte de petróleo bruto. O custo de gerenciamento da parafina é enorme e aumenta significativamente com o aumento da produção de petróleo em áreas marítimas profundas. Mas estes custos podem ser significativamente reduzidos se a deposição de parafina em dutos puder ser prevista com precisão. Nesta pesquisa, o fenômeno de deposição foi analisado numericamente. O modelo de deslizamento foi utilizado para prever o fluxo multifásico e a deposição de parafina foi determinada a partir de um modelo de convecção. Este modelo previu com precisão as taxas de deposição de escala de laboratório em regime de fluxo laminar e turbulento. A taxa de deposição de parafina apresentou uma boa concordância com os resultados do software comercial OLGA. A comparação com a produção de petróleo de um poço real foi modelado, e bons resultados foram obtidos no impacto da queda de pressão devido à redução da área seção transversal causada pela deposição de parafina progressiva na parede do tubo. Os resultados deste trabalho mostraram uma boa consistência física e um acordo razoável com os dados experimentais e de campo comparados.

\section{Palavras-chave}

Deposição de Parafina; Convecção; Escoamento Multifásico; Simulação Numérica; Modelo de Deslizamento. 


\section{Abstract}

Brum, Fabio Paula; Nieckele, Angela Ourivio (Advisor); Stuckenbruck, Sidney (Co-Advisor). Convective Mass Transfer Model to Predict Wax Deposition in Multiphase Flow in Pipelines. Rio de Janeiro, 2014. 111p. MSc. Dissertation - Departamento de Engenharia Mecânica, Pontifícia Universidade Católica do Rio de Janeiro.

Highly waxy crude oils can cause significant operational problems such as blockage of a pipeline due to the precipitation and deposition of select wax components during the production and transportation of the crude oil. The cost of wax management is enormous and rapidly increasing because of increased oil production in deep sea areas. Wax management costs can be significantly reduced if wax deposition in pipeline can be accurately predicted. In this research, the wax deposition phenomenon was numerically investigated. The drift flux model was employed to predict the multiphase flow and the wax deposition was determined based on a convective model. This model accurately predicted the deposition rates for lab scale under laminar and turbulent flows. The wax deposition rate presented a good agreement with the results of commercial software OLGA. A comparison with an existing oil production well was performed, and good results were obtained in the impact in pressure drop due to cross section area reduction caused by progressive wax deposition on the pipe wall. The results of this work showed a good physical consistency and a reasonable agreement with the compared experimental and field data.

\section{Keywords}

Wax Deposition; Convection; Multiphase Flow; Numerical Simulation; Drift Flux Model. 


\section{Sumário}

$\begin{array}{ll}\text { 1. Introdução } & 15\end{array}$

1.1 Revisão Bibliográfica 19

1.2 Objetivo 22

1.3 Organização do Manuscrito 23

2. Modelo Matemático 24

2.1 Equações de Fechamento 27

2.1.1 Transferência de Massa entre as fases 27

2.1.1.1 Massa específica 27

2.1.1.2 Viscosidade dinâmica 31

2.1.2 Tensão cisalhante 32

2.1.3 Fluxo de deslizamento 33

2.1.4 Fluxo de calor 35

2.2 Resistência Térmica na Superfície Interna do Duto 36

2.2.1 Escoamento Monofásico em Dutos de Seção Reta Circular

2.2.2 Escoamento Bifásico em Dutos de Seção Reta Circular

2.3 Modelo de Deposição de Parafina 42

2.3.1 Modelo Difusivo 44

2.3.2 Modelo Convectivo 45

2.4 Gas Lift na Equação de Energia Térmica 48

3. Modelo Numérico 51

3.1 Código TRANSFLUX 52

3.2 Discretização da Equação de Concentração de Parafina 54

3.3 Procedimento da Solução para Deposição de Parafina 56 
4. Comparação com Dados de Laboratório

4.1 Configuração da Seção de Teste 59

4.2 Caracterização do Fluido 61

4.3 Casos Investigados $\quad 63$

4.4 Teste de Malha 63

4.5 Correlações de Transferência de Calor 67

4.6 Comparação entre Modelo Difusivo e Convectivo 75

4.6.1 Escoamento Horizontal 76

4.6.2 Escoamento Levemente Inclinado $\left(2^{\circ}\right)$ - Padrão de Fluxo Intermitente 81

4.6.3 Escoamento Vertical 83

4.7 Análise de Sensibilidade aos Parâmetros de Ajuste de $\begin{array}{ll}\text { Matzain } & 87\end{array}$

5. Resultados de Campo 94

5.1 Geometria 94

5.2 Caracterização do Fluido 95

5.3 Condições de Contorno 96

5.4 Resultados Numéricos $\quad 97$

5.5 Cálculo da Espessura do Depósito 99

5.6 Comparação com Dados de Campo 102

6. Comentários Finais e Sugestões 104

6.1 Comentários Finais 104

$\begin{array}{ll}6.2 \text { Sugestões } & 106\end{array}$

$\begin{array}{ll}\text { 7. Referências Bibliográficas } & 107\end{array}$ 


\section{Lista de Figuras}

Figura 1.1- Produção de Petróleo em Águas Profundas 15

Figura 1.2- Linha de produção parcialmente bloqueada 16

Figura 1.3- Diferentes Tipos de Pigs para Limpeza de Linhas 17

Figura 1.4- Esquema gráfico representativo dos padrões de escoamento

Figura 2.1- Injeção de gas lift em um ponto da coluna de produção

Figura 3.1- Malha de discretização deslocadas 52

Figura 3.2- Fluxograma do Modelo Numérico 58

Figura 4.1- Curva de solubilização de parafina 62

Figura 4.2- Influência da malha na espessura do depósito. Caso 164

Figura 4.3- Influência do passo de tempo na espessura do depósito. Caso 1

Figura 4.4- Influência da malha e passo de tempo na espessura do depósito. Caso 1

Figura 4.5- Mapa de Fluxo gerado pelo Matzain (1999)

Figura 4.6- Influência das correlações de Kudirka et al (1965) e Ravipudi e Godbold (1978). Caso 1, t= 24 horas.

Figura 4.7- Influência das correlações de Shah (1981) e Ravipudi e Godbold (1978), corrigida por Kim et al. (1999). Caso $5, t=24$ horas $(b=0.123)$

Figura 4.8- Influência das correlações de Shah (1981) e Aggour (1978). Caso 8, $\mathrm{t}=24$ horas.

Figura 4.9- Variação temporal da espessura do depósito na extremidade do duto

Figura 4.10- Espessura do depósito na extremidade do duto. Casos 1,2 e 3

Figura 4.11- Perfil de espessura do depósito ao longo do duto. Casos 1,2 e 3. 
Figura 4.14- Espessura do depósito na extremidade do duto. Casos 7, 8 e 9 .

Figura 4.15- Perfil de espessura do depósito ao longo do duto. Casos 7, 8 e 9 .

Figura 4.16- Influência de C2 na espessura de depósito na extremidade final do duto.

Figura 4.17- Influência de C1 e C2 na espessura do depósito na extremidade final do duto.

Figura 4.18- Espessura de depósito na extremidade final comparando os métodos LD-LD e modelo difusivo com o modelo convectivo ajustado

Figura 5.1- Geometria do poço produtor 95

Figura 5.2- Perfil de pressão ao longo da linha sem depósito de parafina

Figura 5.3- Perfil de holdup de líquido ao longo da linha sem depósito de parafina

Figura 5.4- Perfil de temperatura ao longo da linha sem depósito de parafina Influência do passo de tempo na espessura do depósito. Caso 1

Figura 5.5- Perfil de Temperatura próximo ao TDP durante a deposição de parafina

Figura 5.6- Perfil de espessura do depósito de parafina após 5 e 10 dias

Figura 5.7- Evolução com o tempo da espessura de depósito de parafina em três posições diferentes da linha de produção

Figura 5.8- Comparação entre os perfis de espessura de depósito ao longo da linha

Figura 5.9- Perfil de holdup de líquido na região de TDP em diferentes instantes de tempo

Figura 5.10- Variação no tempo da diferença de pressão entre o reservatório e a plataforma 


\section{Lista de Tabelas}

Tabela 2.1 Coeficientes para fator volume de formação 29

Tabela 4.1- Característica do Tubo 59

Tabela 4.2- Característica do Fluido 61

Tabela 4.3- Composição do Fluido 61

Tabela 4.4- Calor específico à pressão constante para os 62 fluidos

Tabela 4.5- Casos Estudados

Tabela 4.6- Correlações de cálculo para $h_{\text {in }}$ conforme literatura atual

Tabela 4.7- Correlações de cálculo para $h_{\text {in }}$ conforme sugestão de Matzain 68

Tabela 4.8- Correlações de cálculo para $h_{\text {in }}$ utilizadas no estudo

Tabela 4.9- Correlações para $h_{\text {in }}$ utilizadas e padrões obtidos em cada caso

Tabela 5.1- Ambiente Externo ao poço 95

Tabela 5.2- $\quad$ Fluido Produzido pelo Reservatório 95

Tabela 5.3- Propriedades Termofísicas dos Fluidos 96 


\section{Lista de símbolos}

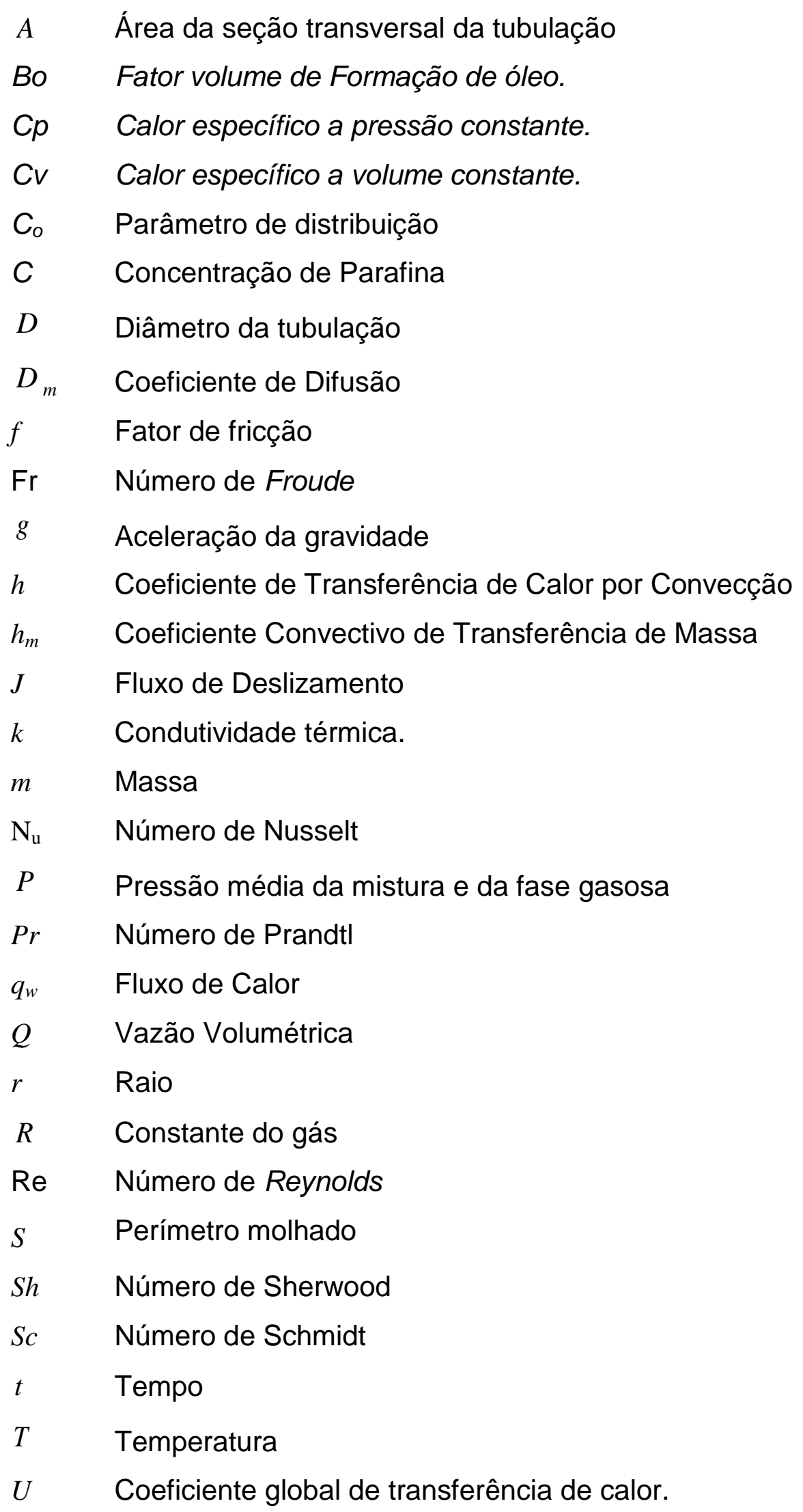




$\begin{array}{ll}u & \text { Energia Interna } \\ v & \text { Velocidade } \\ \dot{\forall} & \text { Vazão volumétrica } \\ x & \text { Coordenada axial } \\ Y & \text { Fator de Expansão } \\ w_{\text {sol }} & \text { Fração da Parafina Saturada }\end{array}$

\section{Símbolos gregos}

$\alpha \quad$ Fração volumétrica da fase

$\Delta \quad$ Variação de uma grandeza

$\mu \quad$ Viscosidade dinâmica

$\varepsilon \quad$ Rugosidade Absoluta.

$v \quad$ Viscosidade cinemática

$\sigma \quad$ Tensão cisalhante

$\delta \quad$ Espessura do Depósito

$\phi \quad$ Porosidade

$\rho \quad$ Massa específica

$\tau \quad$ Tensão de cisalhamento

$\theta \quad$ Ângulo do Escoamento

\section{Subscritos}

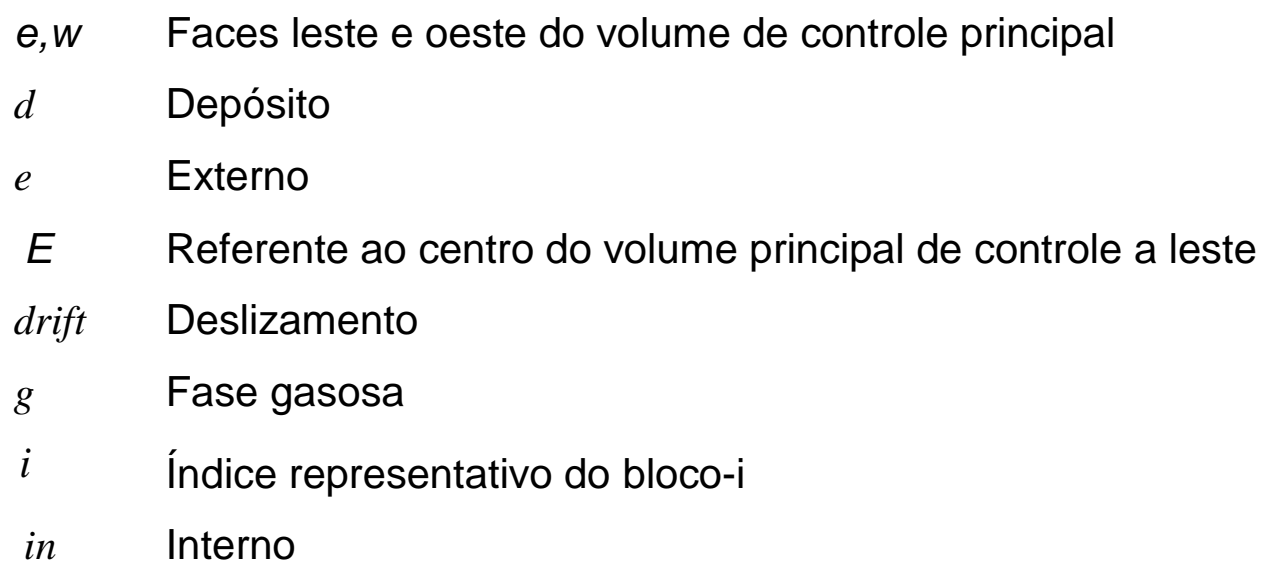


int Interface fluido-parafina

inj Injeção

JT Joule-Thomson

$l \quad$ Fase líquida

$m \quad$ Mistura

$P \quad$ Centro do volume de controle principal

$o \quad$ Fase Óleo

sg Superficial do Gás

sl Superficial do Líquido

$r \quad$ Relativo entre as fases

ref Referência

sol Solubilidade

w Parede da tubulação

wax Parafina

W Corresponde ao volume de controle oeste ao principal

$\infty \quad$ Meio externo

\section{Sobrescritos}

o Referente ao passo de tempo anterior

std Condição Padrão 